\title{
A Robust Palmprint Recognition System Based on Both Principal Lines and Gabor Wavelets
}

\author{
Nouha BEN MAHFOUDH ${ }^{1}$, Yousra BEN JEMAA ${ }^{2}$ and Faouzi BOUCHHIMA ${ }^{3}$ \\ ${ }^{1,3}$ CES Laboratory, ENIS, BP 1173, 3038 Sfax, Tunisia \\ ${ }^{2}$ Signals and systems Unit, ENIT, BP 37, 1002 Tunis, Tunisia \\ yousra.benjemaa@enis.rnu.tn
}

\begin{abstract}
We present in this paper a new palmprint recognition system based on the principal lines of the palm. An original algorithm is proposed in order to detect automatically principal lines and extract their correspondent geometrical features. Given the complexity of the palmprint recognition and in order to ameliorate performances, we propose a hybrid approach based on both geometrical and gabor features.

A comparative study between the three feature vectors obtained from the geometrical approach, global approach and combination of both has proved that the geometrical features are the most relevant since they can give the best compromise recognition Rate/Time. Moreover, a combination of geometrical features with global features can improve recognition rate while keeping the same recognition and learning times. Obtained results also show that the hybrid approach performances are very satisfactory and even surpass the very popular ones.
\end{abstract}

Index Terms - Person recognition, Palmprint, Principal lines, geometrical feature, Gabor wavelets

\section{INTRODUCTION}

The distinctive emergence of data technology in all fields has triggered the need to identify users particularly in security application, access control, forensic etc. As a consequence, automatic people recognition using biometric features attracted wide attention from researchers $[1,2,3]$. One of the biometric features used is the palmprint which is relatively new. Compared with other currently available technologies, the advantages of palmprint recognition are distinctiveness, low-resolution, costeffectiveness, non-intrusiveness and high accuracy [4, 5]. In fact, palmprints contain particular features (principal lines and wrinkles) which can be extracted from low resolution images, having high user acceptability and are considered as stable structure features; the texture of the palm is personal to each individual and lines are unique and persistent for all people over the world and maintained throughout their lives making it useful as a biometric trait for person recognition.

Given these advantages, in recent years, palmprints have been investigated extensively in automated personal authentication. Many techniques for palmprint recogni- tion are developed. They can be classified into three approaches: Line-based approaches, Subspace-based approaches and texture-based approaches.

In the line- based approaches, the features that must be extracted are the lines [6, 7, 8]. L. Zhang and D. Zhang [8] used wavelets to extract the principal lines and wrinkles of the palm. Han and al. [6] used Sobel and morphological operations to extract line-like features from palmprints.

Texture-based approaches are also important for palmprint recognition and have shown good performance in terms of recognition rates [9]. Zhang and al. proposed "PalmCode" method which uses the Gabor filter to extract the texture feature of palmprint [10]. Later, A. Kong and D. Zhang [11] suggested a method called FusionCode that consists of using feature-level fusion strategy, which can be considered as the enhanced version of PalmCode [10].

The research on appearance methods is an active area in pattern recognition field and this technique was also applied to palmprint recognition. It uses Principal Component Analysis (PCA), Linear Discriminant Analysis (LDA) and Independent Component Analysis (ICA) to reduce the size of the features vector already extracted $[12,13,14]$. For example, Xi P and Qi applied the Gabor filter for features extraction and then used (2D) ${ }^{2} \mathrm{PCA}$ to reduce the dimension of the feature vector [12].

Most of these researchers called global approaches use the whole of transformed palmprint image as a feature vector which represent the input of the classification system. This method is efficient in features matching but it is sensitive to illumination and rotation, and it is time consuming in both learning and recognition phase, especially when increasing the number of features.

In this paper, we present a local approach based on the principal lines of the palm in order to extract geometrical features. Local approaches have shown better performance in other biometric recognition systems such as fingerprint or face $[15,16]$. They have the advantage of giving more relevant features than those provided by global approaches while keeping reduced learning and testing times.

The idea in this paper is to merge the two techniques since the combination of geometrical and global features could further improve the recognition rate of the overall approach without increasing the learning and recognition time ensured by the global approaches. 
Four contributions are proposed in this paper. First, we present a new technique to locate the Region of Interest. Secondly, we introduce a new algorithm for extracting the principal lines in order to propose a geometrical descriptor which is very discriminatory despite its reduced size. Thirdly, we propose a hybrid approach based on both principal line features and Gabor features to improve the performance. Finally, the robustness of the approach, especially the use of geometric features is evaluated on the Hong Kong palmprint database.

The rest of this paper is organized as follows. In Section 2, a pre-processing on the palmprint image is described to extract the region of interest ROI. The geometrical features extraction technique is proposed in Section 3. In section 4, the extraction of global features is presented. Experimental results are given in Section 5 proving that our approach gives satisfactory performances thanks to geometrical features and can even surpass the most existing works. Finally, some concluding remarks are given in Section 6.

\section{PALMPRINT PREPROCESSING: ROI EXTRACTION}

ROI extraction is a crucial step and it greatly affects the final recognition results. Most of the existing approaches extract ROI using some key points between fingers [17] making them non robust for fingerless handicap persons.

In order to overcome this limitation, we propose here a new algorithm for extracting ROI with the following steps:

a) Apply a low-pass filter in order to smoothing the original palm image and convert it into a binary map by a thresholding (fig. 1.b)

b) Trace the palm's boundary (fig. 1.c)

c) Use linear searching algorithm, along the palm boundary, to find:

- (L1 and L2) are the two ends of the wrist of the hand

- $\quad$ The key point B is the first intersection of the bisector of the segment [L1 L2] with the contour of the palm

- $\quad$ The key point $A$ is the second intersection of the bisector of the segment [ [ ll L2] with the contour of the hand

- The point $O$ is the midpoint of [AB]

d) The point $\mathrm{O}$ represents the origin of the coordination system and line (AB) is the Y-axis (fig. 1.d).

Finally, a sub-image of fixed size is extracted as a region of interest that will be the surface for further process (fig. 1.e).

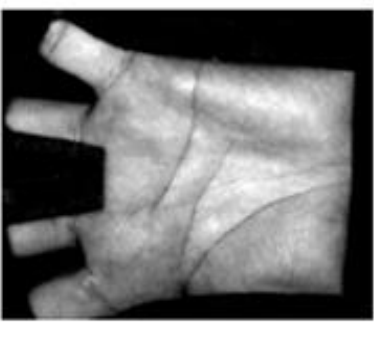

a

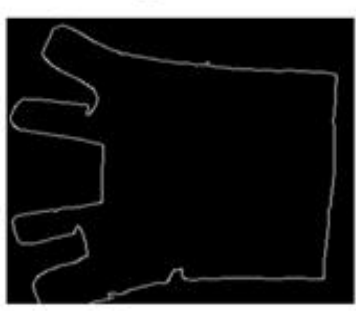

c

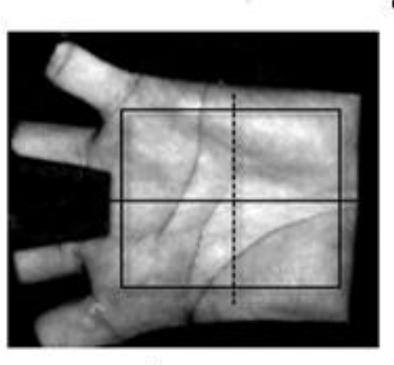

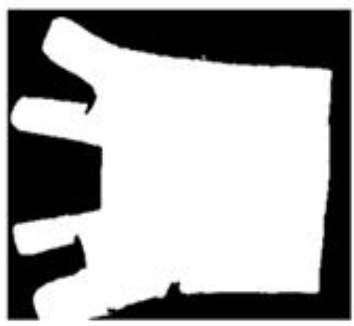

b

d

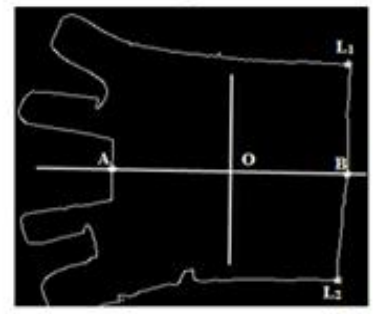

Figure.1 ROI extraction: (a) Original image, (b) Binary image, (c) Contour image, (d) Key points detection, (e) Coordinate system and central part of the palmprint.

\section{GEOMETRICAL FEATURES EXTRACTION}

As we previously mentioned, we used a local approach. This approach focuses on the principal lines of the palm to get geometrical features since they are the most stable and important features in low-resolution images.

\section{A. Principal lines extraction}

Existing techniques for edge detection will extract too many lines from the palmprint.

In this paper, we propose a new algorithm with the following steps:

a) Segmentation

b) Skeletonization

c) Detection of principal lines

\section{Segmentation}

We need here a segmentation step that differentiates the principal lines of the whole texture of the palm. Several segmentation methods exist in the literature. Methods based on regions such as "K Means Clustering" which are very dependent on noise [18]. Methods based on boundaries like the Canny filter which can't provide good performances when the palm texture is very complex. Consequently, we use in this paper, thresholding based methods.

The binarization process depends on the chosen threshold value which should be calculated automatically. 
Since the information of the neighbourhood of pixels is not taken into consideration in a global thresholding, this approach is not adequate for complex textures like palm.

For local binarization, the threshold is adaptive. The value of a pixel does not only depend on the pixel itself but also on its local information or its neighbourhood.

Therefore, thresholding of the image will be done by blocks, the threshold value is fixed by several functions such as the Gaussian function and the average function (see fig. 2).

Adaptive thresholding methods are appreciated. They usually give better results than the method of global thresholding $[19,20]$.

Consequently, we opted to apply binarization on images with an adaptive threshold by using the Gaussian function.

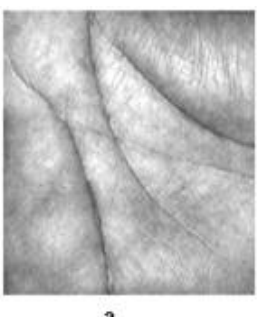

a

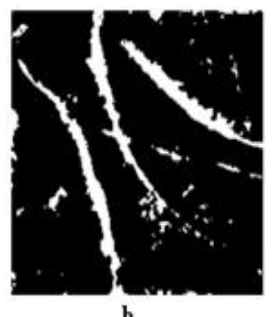

b (a)

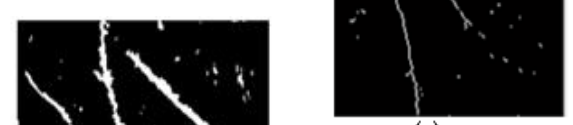

After a skeletonization by successive thinning operations, the image does not only contain the main lines but also some other edges as shown in fig. (3.b).

We propose here a new technique allowing to differentiate only the principal lines.

At first, we classify all white pixels according to the number of their neighbors.

A pixel is considered as isolating if it has no white neighbors, a pixel is considered as termination if it has one neighboring white pixel and finally if the number of white neighbors is more than two, it is about an intersection pixel (See fig. 4).

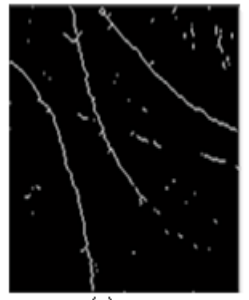

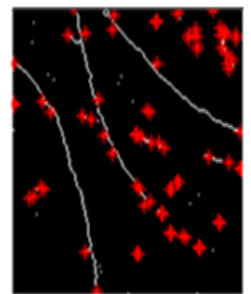

(b)

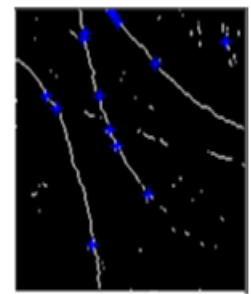

(c)
Figure.4 Classification of pixels: (a) Binary image after Skeletonization,

(b) Detection of end pixels (c) Detection of intersection pixels

Because of the discontinuity of a few lines, a linear interpolation phase is crucial to ensure the continuity of the main lines. This step consists of linking two ending points if the distance between them is very small. For each termination point, we seek the closest termination point to it. If the distance between them is small, we link them. To calculate the distance between the two points, we use the Euclidean distance. Finally after browsing all the ending pixels, we detect all the segments in the image.

We eliminate then the lines of reduced sizes which represent edges of the palm. Therefore, only the principal lines are preserved (See fig. 5).

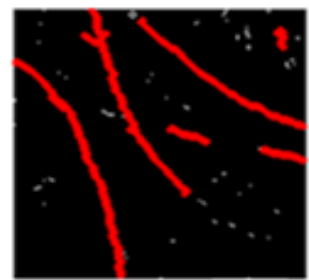

(a)

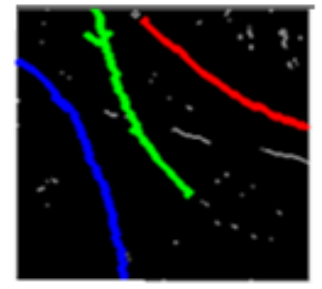

(b) (See Fig. 3).

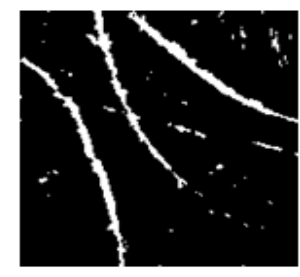

a

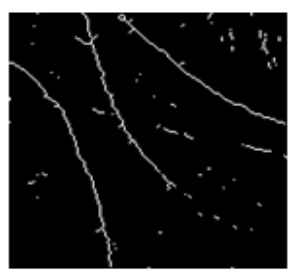

b
Figure. 3 Skeletonization by thinning: (a) original image, (b) Skeletonization of the binary image

\section{Detection of principal lines}

Figure.5 Detection of principal lines: (a) Detection of all lines, (b) De-

\section{B. Feature extraction}

After detection of principal lines and their ends from the palmprint image, some features can be extracted:

- The number of lines which is between 1 and 3 (See Fig. 6).

- The length of each line defined as the distance between the two ends of the principal line using the Euclidean distance.

- The curvature of a line is the longest distance between the line crossing the ends and all points of this line (See Fig.7). tection of principal lines 
Given a line with equation: $\mathrm{D}: \mathrm{ax}+\mathrm{by}+\mathrm{c}=0$ and $\mathrm{a}$ point $\mathrm{M}$ of coordinates $\left(\mathrm{x}_{\mathrm{M}}, \mathrm{y}_{\mathrm{M}}\right)$, the distance between $\mathrm{M}$ and (D) is given as follows:

$$
\mathrm{d}(M, D)=\frac{\left|a^{*} x M+b^{*} y M+c\right|}{\sqrt{a^{2}+b^{2}}}
$$

- The angle formed by two lines

Given two lines D1 and D2 characterized respectively by the two following equations:

$\mathrm{D} 1: \mathrm{y}=\mathrm{ax}+\mathrm{b}$ and $\mathrm{D} 2: \mathrm{y}=\mathrm{a} 1 \mathrm{x}+\mathrm{b} 1$, the angle formed by them is given as follows:

$$
\operatorname{tang}(\varphi)=\frac{a_{2}-a}{1+a^{*} a_{2}}
$$

Hence, the feature vector has ten values which are: the number of main lines, the length of each line, the curvature of each line and the angles formed by two lines as shown in fig. 8 . Any missing value will be zero.

Thus, if the palm has two principal lines, so the first value is equal to 2 , fourth, seventh, ninth and the last value of the vector are all equal to 0 .

If the palm has a single main line, so the first value is equal to 1 , the third, fourth, sixth, seventh, eighth, ninth and the last value of the vector are all equal to 0 .

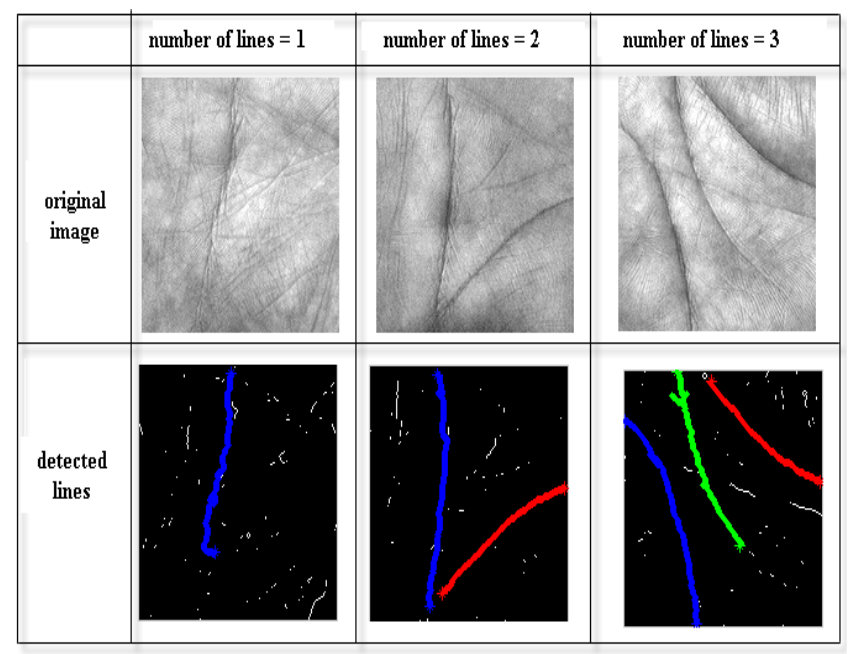

Figure.6 Detecting lines of each palmprint

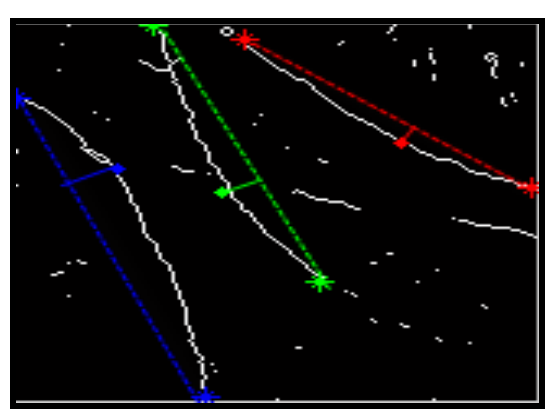

Figure.7 Determination of the curvature of principal lines

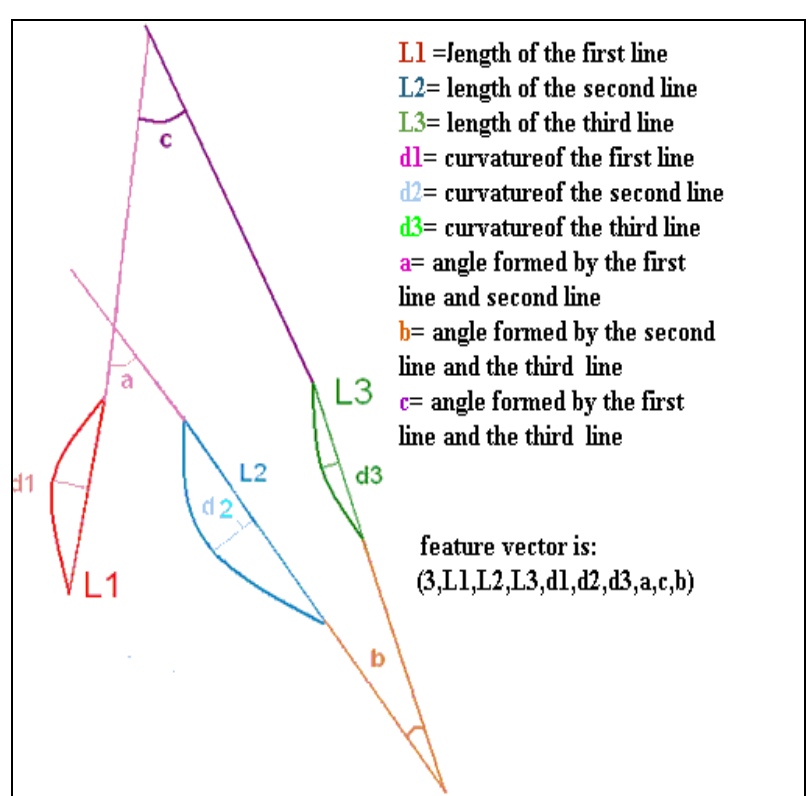

Figure. 8 Feature vector, example of palm having three principal lines

\section{IV.EXTRACTION OF GLOBAL FEATURES}

To further improve the efficiency of the whole palmprint recognition system, we use, in joint with geometrical characteristics, Gabor features. Gabor filters have shown a high efficiency in various applications. Also, known as a precise localization in time/frequency and in robustness to changes in contrast and brightness, it is qualified to extract the frequency content of the oriented images. Our approach is based on 2D Gabor filtering represented by the following equation used in [22]:

$$
\mathrm{G}_{(u, v)}^{(z)}=\frac{\left\|k_{u, v}\right\|^{2}}{\sigma^{2}} e\left(-\left\|k_{u, v}\right\|^{2}\|z\|^{2} / 2 \sigma^{2}\right)\left[e^{i k u, v z}-e \frac{\sigma^{2}}{2}\right]
$$

With

$k_{u, v}=\left(\begin{array}{l}k_{f x} \\ k_{f y}\end{array}\right)=\left(\begin{array}{l}k_{v} \cos \phi_{u} \\ k_{v} \sin \phi_{u}\end{array}\right)$

$\mathrm{k}_{\mathrm{v}}=f_{\max } / 2^{\mathrm{v} / 2}, v$ : frequency, $f_{\max =\pi}, \varnothing_{u}=u(\pi / 8), u$ : orientation, $\sigma$ : the standard deviation of the Gaussian and $\mathrm{z}=(\mathrm{x}, \mathrm{y})$

An obvious dimension reduction consists of applying to the vectors already extracted, the bi-dimensional Principal Component Analysis (2DPCA). It has a high performance in terms of recognition, computation time and reconstruction of the original images compared to ICA, LDA and PCA [13]. Fig. 9 shows the convolution of a palmprint image by six Gabor filters at different orientations and frequencies. 


\section{EXPERIMENTAL RESULTS AND COMPARATIVE STUDY}

To classify or compare the palms that are already represented by feature vectors, we need a matching method or a classifier. For experimentation, we use a neural networks classifier. In our study, we have used three types of features:

- Geometrical features

- Gabor features

- Combined information between Gabor and geometrical features

\section{A. Palmprint database}

Tests are made on the Hong Kong Polytechnic University open palmprint database [23]. It contains 7752 grayscale images corresponding to 386 different palms. These images are of size $384 \times 284$ pixels and BMP format.

Twenty samples of palms were collected in two sessions: ten samples were collected in the first session and the other in the second session. The interval average between the two collections was of 2 months.

The database is split into subsets at random: $30 \%$ of the database was used for training and $70 \%$ were acquired for testing process. All tests are made on five random combinations of palmprint samples.

\section{B. Experimental results and discussion}

Experimental results are shown in table1. Particularly, we give the average recognition rate and time using respectively Gabor features (Global approach), geometrical features (Local approach) and the fusion of them (Hybrid approach).

From table 1 and fig. 10, we can notice that:

- The hybrid approach ensures the best recognition rate because it takes advantages of the merits of Gabor wavelets and geometrical features.

- The recognition rate of the local approach is satisfactory (about 77\%) but it is lower than the global approach which uses 500 features considered extremely large compared to the ten geometrical features.

- The learning and testing times of the global approach are very large proving its inapplicability in real time context.

- The experimental results show that the geometrical features are more relevant than global features and can greatly improves the recognition rate without increasing the learning time when adding to the Gabor features. Thus, they can work in real time context.
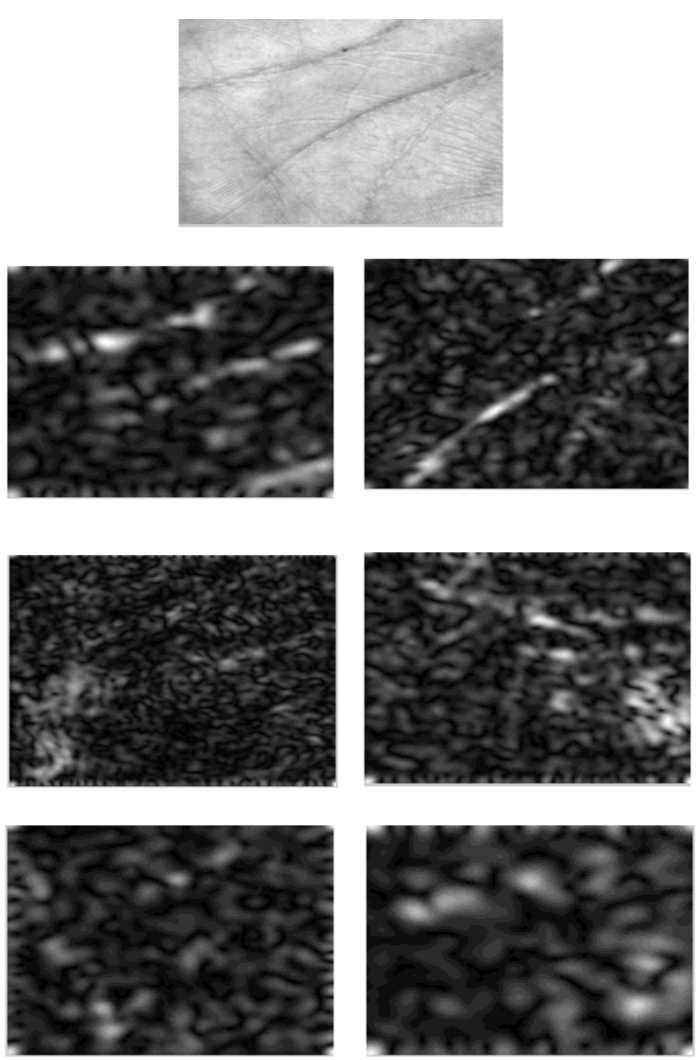

Figure.9 Palmprint Gabor features

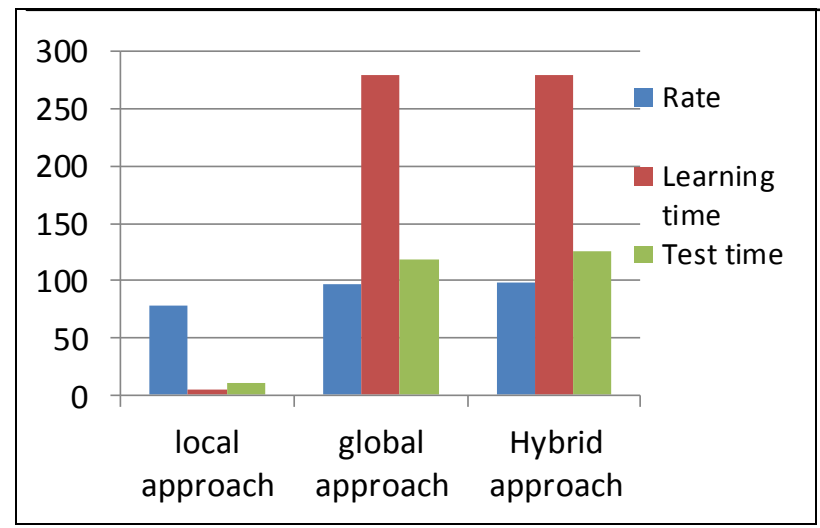

Figure.10 Compromise Rate / Time recognition for all approaches

In fact, fig. 11 shows that the learning time of the local approach is incomparable with that of the global approach and hybrid approach for all number of Gabor filters.

Also, the learning time of the hybrid approach is very similar to the global approach. Thus, for a real-time application, it is better to add more geometrical features to improve the recognition rate by keeping a short learning time. 


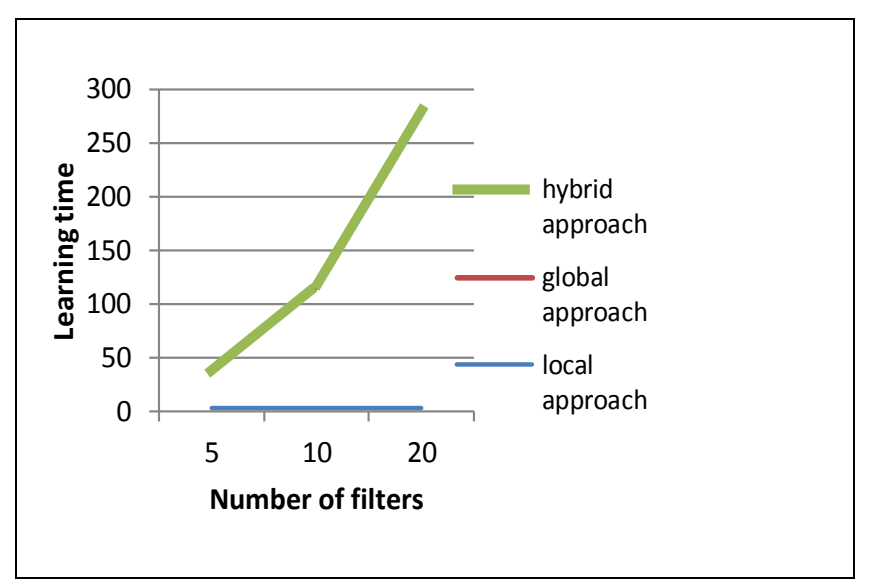

Figure.11 Learning time versus the number of Gabor filters for all approaches

\section{Comparison with other approaches}

To enhance the performance of our proposed approach, we present a comparative study with two existing works.

The first approach [10] proposed by W. K Kong, D. Zhang and W. Li extract Gabor features. Steps of the proposed approach are as follows:

- Image acquisition: Images are captured by a scanner of palms and the captured images are converted into digital images for further processing.

- Preprocessing: extracting the central part of the palm using the limits of the fingers.

- Features extraction using a Gabor filters applied on the central part of the palm.

- Identification: a distance function is used to measure the similarity between images.

Xi P and Qiu-Qi [12] used Gabor features for recognition with a dimensionality reduction thanks to the 2DPCA and the Euclidean distance and the KPPV classifier for the classification step.

All tests for all approaches are made in the Hong Kong Palmprint database. Table2 represents the recognition rates and the number of features for all compared approaches.

We can notice that the proposed approach (hybrid approach) provides the best performances compared to the other works. This proves that the used features which some of them are geometric are the most representative in terms of people recognition by palmprint.

The computation time, which is proportional to the size of the feature vector, represents a very important criterion to evaluate the reliability of a system. As the local proposed approach uses a very small number of features, it can be applied in real time context. Furthermore, the addition of other geometrical features can improve the recognition rate by keeping a very low learning time.

Thus, the use of geometrical features can ensure the best compromise rate/time recognition compared to the most existing approaches and therefore it is always the best solution for real-time application.

\section{VI.CONCLUSION AND FUTURE WORKS}

We have proposed in this paper a novel hybrid approach for palmprint recognition by using both principal line based features and Gabor features.

Our geometric approach has a reduced vector size features compared to the other approaches and generates a very satisfactory recognition rate similar to these of the other approaches. So the geometrical features are more suitable compared to those given by the global approaches. Consequently, the addition of other geometrical features can improve the recognition rate by keeping very short learning times. Thus, the use of geometric features can ensure the best compromise Rate / Time recognition, and it is always the best solution for a real time application.

Several perspectives can be considered. We can improve our local approach by adding other geometrical features, as they are relevant, like the number of intersections of the lines.

We can use decision trees for classification as they are suitable in our context. Decision trees provide a hierarchical classification and support the heterogeneous data and nonlinear effects very well. The idea is to use at the beginning only geometrical features for sub-classification and then using global features to classify only the subsets already found. This allows to have the same given rate by the hybrid approach while keeping a low learning and testing times.

TABLE.1 COMPARISON OF THE THREE APPROACHES

\begin{tabular}{|c|c|c|c|}
\hline Approach & Recognition Rate (\%) & Recognition time(sec) & Learning time(sec) \\
\hline Global & 98.8 & 117.19 & 281.14 \\
\hline Local & 76.74 & 03.40 & 10.8 \\
\hline Hybrid & 99.22 & 128.07 & 283.26 \\
\hline
\end{tabular}


TABLE.2 COMPARISON BETWEEN ALL APPROACHES

\begin{tabular}{|c|c|c|c|}
\hline Approach & $\begin{array}{c}\text { Number of samples } \\
\text { (Learning/test) }\end{array}$ & $\begin{array}{c}\text { Size of feature vec- } \\
\text { tor }\end{array}$ & Recognition Rate (\%) \\
\hline Approach [8] & $5 / 5$ & $64 \times 64 \times 12$ & 92.88 \\
\hline Approach [12] & $5 / 5$ & $32 \times 30 \times 12$ & 99 \\
\hline Global approach & $3 / 7$ & $5 \times 5 \times 20$ & 98.83 \\
\hline Hybrid approach & $3 / 7$ & $5 \times 5 \times 20+10$ & 76.74 \\
\hline Geometric approach & $3 / 7$ & 10 & 99.22 \\
\hline
\end{tabular}

\section{REFERENCES}

[1] Gorodnichy, D.O., "Multi-order biometric score analysis framework and its application to designing and evaluat-ing biometric systems for access and border control", IEEE Workshop on Computational Intelligence in Bio-metrics and Identity Management (CIBIM), pp $44-53$ (2011)

[2] Monwar, M.M, "A novel fuzzy multimodal informationfusion technology for human biometric traits identifica-tion", International Conference on Cognitive Informatics \& Cognitive Computing (ICCI*CC ), pp 112 - 119, Au-gust (2011)

[3] Y. Ben Jemaa, A. Derbel and A. B. Jmâa, "2DPCA frac-tal features and genetic algorithm for efficient face representation and recognition", Eurasip journal on Information Security, Vol. 2011, N. 1(2011)

[4] D. S. Huang, W. Jia, and D. Zhang, "Palmprint verifica-tion based on principal lines", Pattern Recognition, Vol.41, pp. 1316-1328, April (2008)

[5] A. Kongo, D. Zhang and M. Kamel, "A survey of palmprint recognition”, Pattern Recognition, Vol.42, pp. 1408-1418 (2009)

[6] C.Han, H.Cheng, C.Lin and K.C. Fan, "Personal authen-tication using palmprint features", Pattern Recognition, Vol.36, N.2, pp. 371-381 (2003)

[7] Rotinwa-Akinbile, M.O.Aibinu and A.M. Salami, "Palmprint Recognition Using Principal Lines Characteri-zation", International Conference on Informatics and Computational Intelligence (ICI), pp. 278282, December (2011)

[8] L.Zhang and D.Zhang, "Characterization of Palmprints by Wavelet Signatures via Directional Context Model-ing", IEEE Trans. on SMC - B, Vol.34, N.3, pp. 1335-1347, June (2004)

[9] Yan-Xia Wang and Guang-Hua Sun, "Palmprint recog-nition using Palm-line direction field texture feature", International Conference on Machine Learning and Cybernetics (ICMLC), Vol. 3, pp. 1130-1134, July (2012)

[10] D. Zhang, A. Kong, J. You and M. Wong, "Online palmprint identification", IEEE Trans. Pattern. Anal. Mach. Intell. Vol.25, N.9, pp. 1041-1050 (2004)

[11] A. Kong, D. Zhang and M. Kamel, "Palmprint identific-ation using feature-level fusion", Pattern Recognition, Vol. 39, pp. 478-487 (2006)

[12] X. Pan, and Q.-Q. Ruan, "Palmprint recognition using Gabor feature-based (2D)2PCA", Neurocomputing, Vol. 71, pp. 3032-3036 (2008)
[13] Zhong-Qiu, De-Shuang and Wei Jia, "Palmprint recog-nition with 2DPCA+PCA based on modular neural net-works" Neurocomputing, Vol. 71, pp. 448-454 (2007)

[14] Xin Pan and Q.Ruan, "Palmprint recognition with im-proved two-dimensional locality preserving project-tions", Image Vision Comput. Vol. 26, N.9, pp. 261-1268 (2008)

[15] Qingqing Fu, Aiping Wu and Yonghua Li, "Fingerprint Identification System Based on SOPC", International Conference on Wireless Communications, Networking and Mobile Computing (WiCOM), pp.1 - 4, September (2011)

[16] Y. Ben Jemaa and S. Khanfir, "Automatic local Gabor Features extraction for face recognition", International journal on Computer Science and Information Security, Vol. 3, N. 1, pp. 116-122 (2009)

[17] G. K. Michael, T. Connie and A. B. J. Teoh, "Touchless palmprint biometrics: Novel design and implementa-tion", Image Vision Comput, Vol. 26, N.12, pp. 1551- 1560, December (2008)

[18] Sulaiman, S.N.and Isa, N.A.M., "Adaptive fuzzy-Kmeans clustering algorithm for image segmentation", IEEE Transactions on Consumer Electronics, Vol. 56, N.4, pp. 2661 - 2668, November (2010)

[19] N. Otsu, "A thresholding selection method from gray-level histograms", IEEE Trans. Sys, Man and Cyb, Vol.9, N.1, pp. 62-66

[20] Kanungo, P.Nanda, P.K. and Ghosh, A., "Parallel genetic algorithm based adaptive thresholding for image seg-mentation under uneven lighting conditions", Interna-tional Conference on Systems Man and Cybernetics (SMC), pp. 1904-1911, October (2010)

[21] Srijeyanthan K., Thusyanthan A., Joseph C.N., Kokulakumaran S., Gunasekara C. and Gamage C., "Skeletonization in a real-time gesture recognition sys-tem", International Conference on Information and Au-tomation for Sustainability (ICIAFs), pp. 213 - 218, December (2010)

[22] B. Z, S. Sh, X. Ch and W. Gao, " Histogram of Gabor Phase Patterns (HGPP): A Novel Object Representation Approach for Face Recognition", IEEE Trans. on Image Processing, Vol.16, N.1, pp.57-68 (2007)

[23] PolyU Palmprint Database, http://www4.comp.polyu.edu.hk/ biometrics/ 
Nouha BEN MAHFOUDH was born in Tunisia. He received the electrical engineering degree from Faculty of Sciences of Sfax and the master in Computer science from the National Engineers School of Sfax. She is actually a PHD student.

Yousra BEN JEMAA was born in Tunisia. She received a Ph.D. in Electrical Engineering from the National Engineers School of Tunis (ENIT) in 2003. In June 2012, she obtained the HDR in Telecommunications from the National Engineers School of Tunis, University El Manar, TUNISIA.

She joined National Engineers School of Sfax (ENIS) in 2000 where she is an associate Professor in Telecommunication Engineering.

She is a member of the research Lab. U2S at ENIT.

Faouzi BOUCHHIMA was born in Tunisia. He received the electrical engineering degree and the master and the Ph.D degree in industrial computer from the National Engineers School of Sfax, Tunisia.

From 2007, he was a research associate with the Computer Embedded System (CES) Laboratory. He is currently an assistant professor at the Gabes University. 